Bull. Austral. Math. Soc.

16D70, 16P70, 19D80, 16D90

VOL. 52 (1995) [107-116]

\title{
RINGS CHARACTERISED BY SEMIPRIMITIVE MODULES
}

\author{
Yasuyuki Hirano, Dinh van Huynh and Jae Keol Park
}

\begin{abstract}
A module $M$ is called a CS-module if every submodule of $M$ is essential in a direct summand of $M$. It is shown that a ring $R$ is semilocal if and only if every semiprimitive right $R$-module is CS. Furthermore, it is also shown that the following statements are equivalent for a ring $R$ : (i) $R$ is semiprimary and every right (or left) $R$-module is injective; (ii) every countably generated semiprimitive right $R$-module is a direct sum of a projective module and an injective module.
\end{abstract}

\section{INTRODUCTION}

Let $R$ be a ring and $M$ be a right $R$-module. Then $M$ is called a semiprimitive module if the Jacobson radical of $M$ is zero. If every semiprimitive right module over a ring $R$ is injective, then $R$ is a semisimple ring by [8]. However if we weaken injectivity to quasi-injectivity then we only obtain a characterisation of semilocal rings which is quite far from being semisimple. We prove the following:

THEOREM 1. For a ring $R$ the following conditions are equivalent:

(a) Every semiprimitive right $R$-module is quasi-injective.

(b) Every semiprimitive right $R$-module is $C S$.

(c) Every 2-generated semiprimitive right $R$-module is quasi-continuous.

(d) $R$ is a semilocal ring.

(e) The left-handed version of any one of (a), (b) and (c).

A ring $R$ is called a right (left) $S I$-ring if every singular right (left) $R$-module is injective. In [4] it is shown that a ring $R$ is right Artinian, right and left SI if every countably generated right $R$-module is a direct sum of a projective module and an injective module. Motivated by this we establish the following theorem:

ThEOREM 2. For a ring $R$ the following conditions are equivalent:

(i) Every semiprimitive right $R$-module is a direct sum of a projective module and an injective module.

Received 20th September, 1994

Dinh van Huynh and Jae Keol Park were supported by the KOSEF, TGRC and the Basic Science Research Institute Program, Ministry of Education, Korea in 1994, Project No. BSRI-94-1402.

Copyright Clearance Centre, Inc. Serial-fee code: 0004-9729/95 \$A2.00+0.00. 
(ii) Every countably generated semiprimitive right $R$-module is a direct sum of a projective module and an injective module.

(iii) $R$ is a semiprimary right and left SI-ring.

(iv) The left-handed version of either (i) or (ii).

We note that if every 2-generated right $R$-module is quasi-continuous, then in particular, for each cyclic $R$-module $X, X \oplus R$ is quasi-continuous and hence $X$ is injective by $[9$, Proposition 2.10] and therefore $R$ is semisimple by [10, Theorem]. Also we observe that if the hypothesis in (c) of Theorem 1 is weakened to cyclic semiprimitive right $R$-modules then the ring $R$ is not necessarily semilocal. For example, considering the ring $\mathbb{Z}$ of integers we see that even each cyclic $\mathbb{Z}$-module is quasi-continuous. However $\mathbb{Z}$ is not (semi)local. For a detailed study of rings (respectively, finitely generated quasi-projective modules) whose cyclic modules (respectively, factor modules) are quasi-continuous we refer to $[6,12]$ and authors cited therein.

\section{Preliminaries}

Throughout this paper we consider associative rings with identity and all modules are unitary. For a module $M$ we write $M_{R}$ (respectively, ${ }_{R} M$ ) to indicate that $M$ is a right (respectively, left) $R$-module where $R$ is a ring. The socle and the Jacobson radical of $M$ are denoted by Soc $(M)$ and $J(M)$, respectively. Let $M$ and $N$ be modules and $I$ be an index set. Then $M^{(I)}$ denotes the direct sum of $|I|$ copies of $M$, and $N$ is called $M$-generated if there exist an index set, say $I$, and an epimorphism from $M^{(I)}$ to $N$.

A module $M$ is called semisimple if $M=\operatorname{Soc}(M)$. For a ring $R, R$ is said to be a semisimple ring if $R=\operatorname{Soc}\left(R_{R}\right)$, or equivalently if $R=\operatorname{Soc}\left({ }_{R} R\right)$. A ring $R$ is called semilocal if $R / J(R)$ is semisimple. If $R$ is semilocal and $J(R)$ is nilpotent then $R$ is said to be semiprimary.

A submodule $E$ of a module $M$ is called an essential submodule of $M$ if $E \cap U \neq 0$ for each non-zero submodule $U$ of $M$. By definition, the singular submodule of $M_{R}$ is the following set:

$$
Z\left(M_{R}\right)=\{a \in M \mid a K=0 \text { for some essential right ideal } K \text { of } R\} .
$$

If $Z(M)=M, M$ is called a singular module. By [3] a module $M$ is singular if and only if there exists a module $A$ containing an essential submodule $B$ such that $M \cong A / B$. By the definition we also see that a non-zero singular module does not contain non-zero projective submodules. In case $Z(M)=0, M$ is called a non-singular module. A ring $R$ is called right non-singular if $Z\left(R_{R}\right)=0$.

Following Goodearl [3] we call a ring $R$ right (left) $S I$ if every singular right (left) $R$-module is injective. The structure of right SI-rings is obtained in [3, Theorem 3.1] 
as follows: $A$ ring $R$ is right $S I$ if and only if $R$ is right non-singular and has a ring direct sum decomposition $R=K \oplus R_{1} \oplus \cdots \oplus R_{n}$ where $K / \operatorname{Soc}\left(K_{K}\right)$ is semisimple and each $R_{i}$ is Morita-equivalent to a right SI-domain.

The Jacobson radical $J(M)$ of a module $M$ is the intersection of all maximal submodules of $M$. A module $M$ is called semiprimitive if $J(M)=0$. If $R$ is a right $V$-ring, that is, every simple right $R$-module is injective, then every right $R$-module is semiprimitive (see [8]). From this fact and Osofsky's result in [11] we easily see that a ring $R$ is semisimple if and only if every cyclic semiprimitive right $R$-module is injective.

A module $M_{R}$ is defined to be a $C S$-module if each submodule of $M$ is contained as an essential submodule in a direct summand of $M$. A ring $R$ is called a right CS-ring if $R_{R}$ is a CS-module. Let $M$ be a module and $N$ be any submodule of $M$. Then by Zorn's Lemma $N$ has a maximal essential extension $N^{*}$ in $M$, that is, $N^{*}$ is a submodule of $M$ which is maximal with respect to the condition that $N \subseteq N^{*}$ and $N$ is essential in $N^{*}$. If $M$ is CS, then $N^{*}$ is a direct summand of $M$. Recently CSmodules have been studied extensively. We refer to [10] and [12] to show how useful this concept is.

Finally a CS-module $M_{R}$ is called quasi-continuous if for any two direct summands $M_{1}$ and $M_{2}$ of $M$ also $M_{1} \oplus M_{2}$ is a direct summand of $M$ whenever $M_{1} \cap M_{2}=0$.

For general background we refer to the texts by Anderson and Fuller [1], Faith [2], Goodearl [3], Mohamed and Müller [9] and Wisbauer [13].

\section{The Proofs}

First we prove Theorem 1.

The implications $(a) \Rightarrow(b)$ and $(a) \Rightarrow(c)$ are clear.

(b) $\Rightarrow$ (d). Assume (b). Put $\bar{R}=R / J(R)$. Then $\bar{R}_{R}$ and $\bar{R}_{\bar{R}}$ are semiprimitive modules. Clearly $\bar{R}$ satisfies (b), too. Hence for each index set $I, \bar{R}_{\bar{R}}^{(I)}$ is a CS right $\bar{R}$-module. Now let $M$ be an arbitrary right $\bar{R}$-module. Then there exist an index set $I$ and an epimorphism $\varphi$ from $\bar{R}_{\bar{R}}^{(I)}$ onto $M_{\bar{R}}$. Since $\bar{R}_{\bar{R}}^{(I)}$ is CS, we have

$$
\bar{R}_{\bar{R}}^{(I)}=\bar{A} \oplus \bar{B}
$$

with $\operatorname{ker}(\varphi) \subseteq \bar{A}$ and $\operatorname{ker}(\varphi)$ is essential in $\bar{A}$, so $\bar{A} / \operatorname{ker}(\varphi)$ is singular. Hence the isomorphism

$$
M \cong \bar{R}_{\bar{R}}^{(I)} / \operatorname{ker}(\varphi) \cong \bar{A} / \operatorname{ker}(\varphi) \oplus \bar{B}
$$

shows that $M$ is a direct sum of a singular module and a projective module. Thus by [10, Theorem 3.18], $\bar{R}$ is semisimple, proving (d). 
(c) $\Rightarrow$ (d). Assume (c). Put $\bar{R}=R / J(R)$. Let $X$ be a simple right $\bar{R}$-module. Then $X \oplus \bar{R}$ is a 2-generated semiprimitive right $R$-module. By (c), $X \oplus \bar{R}$ is quasicontinuous and hence $X$ is $\bar{R}$-injective by [9, Proposition 2.10]. It follows that $\bar{R}$ is a right $\mathrm{V}$-ring and so every right $\bar{R}$-module is semiprimitive (see [8]). Now let $Y$ be a cyclic right $\bar{R}$-module, that is, there exists a right ideal $A$ of $R$ containing $J(R)$ such that $Y \cong R / A$. Then $Y$ is a semiprimitive right $R$-module. By (c), the right $R$-module $Y \oplus \bar{R}$ is quasi-continuous and hence $Y_{R}$ is $\bar{R}_{R}$-injective. Therefore $Y_{\bar{R}}$ is injective. This means that every cyclic right $\bar{R}$-module is $\bar{R}$-injective. By [11], $\bar{R}$ is semisimple, proving (d).

(d) $\Rightarrow$ (a). Assume (d). Let $M$ be a semiprimitive right $R$-module. Then $M J(R)=$ 0 , hence $M$ is also a right $\bar{R}$-module, where $\bar{R}=R / J(R)$. Thus $M$ is a direct sum of simple right $\bar{R}$-modules. But every simple right $\bar{R}$-module is also a simple right module over $R$. Therefore $M_{R}$ is semisimple and so $M_{R}$ is quasi-injective, proving (a).

(d) $\Leftrightarrow(e)$ is clear by the symmetry of (d).

The proof of Theorem 1 is complete.

To prove Theorem 2 we consider a more general situation, namely we study SImodules $M$ via the corresponding category $\sigma[M]$. For a ring $R$ we denote by Mod- $R$ the category of all right $R$-modules. Let $M$ be a right $R$-module. Following Wisbauer $[13]$ we denote by $\sigma[M]$ the full subcategory of Mod- $R$ whose objects are submodules of $M$-generated modules. We fix the module $M$ and define $M$-singularity and $M$ nonsingularity in $\sigma[M]$ as follows.

Let $N$ be a right $R$-module. Then $N$ is called singular in $\sigma[M]$, or simply, $M$ singular if there is a module $K$ in $\sigma[M]$ which contains an essential submodule $L$ such that $N \cong K / L$. By this definition every $M$-singular right $R$-module belongs to $\sigma[M]$. For $M=R$ the notion $R$-singularity is identical to the usual definition of singular modules in Mod- $R$ given in Section 2.

The class of $M$-singular modules is closed under submodules, homomorphic images and direct sums (see $[13,17.3$ and 17.4]). Hence every module $N \in \sigma[M]$ contains a largest $M$-singular submodule, which we denote by $Z_{M}(N)$. If $Z_{M}(N)=0, N$ is called $M$-nonsingular, or nonsingular in $\sigma[M]$. A module $M$ is called hereditary in $\sigma[M]$ if every submodule of $M$ is projective in $\sigma[M]$ (see $[13,39.1]$ ).

Following [5], we call a module $M$ an $S I$-module if every $M$-singular module is $M$-injective. Basic facts about SI-modules can be found in [5]. We begin with the following lemma. (Parts (ii), (iii) and (iv) are known however we include the proof of these here for the sake of completeness.)

Lemma 3. For a quasi-projective right $R$-module $M$ the following conditions are equivalent: 
(i) Every cyclic semiprimitive $M$-singular module is $M$-injective.

(ii) $Z_{M}(M)=0$ and each $M$-singular module is semisimple.

(iii) $M$ is an SI-module.

If $M$ is projective in $\sigma[M]$ then (i), (ii) and (iii) are equivalent to:

(iv) $M$ is hereditary in $\sigma[M]$ and each $M$-singular module is semisimple.

Proof: (i) $\Rightarrow$ (ii). Assume (i). Then in particular, every simple $M$-singular module is $M$-injective. Now let $X$ be a cyclic $M$-singular module. Using an argument in [8] we first verify that $X$ is semiprimitive. Let $0 \neq x \in X$. Then by Zorn's Lemma there exists a submodule $U$ of $X$ which is maximal with respect to $x \notin U$. Hence $(x R+U) / U$ is simple, so by (i), $(x R+U) / U$ is $M$-injective. It follows that there exists of a submodule $V$ of $X$ containing $U$ such that

$$
X / U=(x R+U) / U \oplus V / U
$$

If $V \neq U$, then $x \in V$, a contradiction. Hence $V=U$, which shows that $U$ is a maximal submodule of $X$. From this we infer that $J(X)=0$. Hence by (i), $X$ is $M$-injective. Moreover, it follows that every cyclic submodule of any factor module of $X$ is also $M$-injective. From this and [1, Proposition 16.13] it follows that every cyclic submodule of any factor module of $X$ is $X$-injective. Hence by using [12, Theorem 1] we see that $X$ is semisimple. This implies that every $M$-singular module is semisimple.

If $Z_{M}(M) \neq 0$, then by the previous argument, $M$ contains a minimal $M$-injective $M$-singular submodule $S$. However $S$ is then a direct summand of $M$ and so $S$ is $M$ projective, which is a contradiction. Hence $Z_{M}(M)=0$, proving (ii).

(ii) $\Rightarrow$ (iii). Assume (ii). Let $N$ be an $M$-singular module and $\varphi$ be a homomorphism from a submodule $E$ of $M$ to $N$. Without loss of generality we may assume that $E$ is essential in $M$. Since $E / \operatorname{ker}(\varphi)$ is isomorphic to a submodule of $N$ and $M$ is $M$-nonsingular by (ii), we easily check that $\operatorname{ker}(\varphi)$ is essential in $E$ and hence $\operatorname{ker}(\varphi)$ is essential in $M$. By (ii), $M / \operatorname{ker}(\varphi)$ is then a semisimple module. Hence

$$
M / k e r(\varphi)=E / \operatorname{ker}(\varphi) \oplus A / \operatorname{ker}(\varphi)
$$

for some submodule $A$ of $M$ containing $\operatorname{ker}(\varphi)$. From this we easily see that $\varphi$ can be extended to a homomorphism from $M$ to $N$, proving the $M$-injectivity of $N$. Thus $M$ is an SI-module.

(iii) $\Rightarrow$ (i) is obvious.

Now if $M$ is projective in $\sigma[M]$, then we prove the following:

(iii) $\Rightarrow$ (iv). Assume (iii). Let $N$ be an arbitrary submodule of an $M$-injective module $Q$ in $\sigma[M]$ and denote by $E(N)$ the $M$-injective hull of $N$ in $Q$ with $N \subseteq$ 
$E(N)$. Then $Q=E(N) \oplus Q^{\prime}$ for some $M$-injective submodule $Q^{\prime}$ of $Q$. Moreover, $E(N) / N$ is $M$-singular and therefore $M$-injective. Thus the isomorphism

$$
Q / N \cong E(N) / N \oplus Q^{\prime}
$$

shows that $Q / N$ is $M$-injective. By $[13,39.6], M$ is then a hereditary module in $\sigma[M]$. The fact that every $M$-singular module is semisimple can be proved as in the case (i) $\Rightarrow$ (ii).

(iv) $\Rightarrow$ (ii) is clear.

The proof of Lemma 3 is complete.

ThEOREM 4. For a quasi-projective right $R$-module $M$ the following conditions are equivalent:

(a) Every semiprimitive module in $\sigma[M]$ is a direct sum of an $M$-projective module and an $M$-injective module.

(b) Every countably generated semiprimitive module in $\sigma[M]$ is a direct sum of an $M$-projective module and an $M$-injective module.

(c) $M$ is an SI-module such that $M / \operatorname{Soc}(M)$ is semisimple and every semiprimitive module in $\sigma[M]$ is semisimple.

ProOF: $(a) \Rightarrow(b)$ is obvious.

(b) $\Rightarrow$ (c). Assume (b). Then every cyclic semiprimitive $M$-singular module must be $M$-injective. By Lemma 3, $M$ is then an SI-module.

Now let $S=\operatorname{Soc}(M)$. Assume that $S$ is not essential in $M$. Then there exists a non-zero finitely generated submodule $W$ of $M$ such that $S \cap W=0$. Hence $\operatorname{Soc}(W)=0$. Therefore the same argument as in the first part of proving (i) $\Rightarrow$ (ii) (Lemma 3) shows that $W$ is semiprimitive. Then by (b), $W$ contains a non-zero $M$ projective direct summand $U$ which is in particular finitely generated, quasi-projective and $\operatorname{Soc}(U)=0$. Since the object set of $\sigma[M]$ is closed under direct sums, homomorphic images and subobjects, it follows that $\sigma[U]$ is a subcategory of $\sigma[M]$. Hence, if $Y$ is a cyclic semiprimitive $U$-singular module, then $Y$ is also $M$-singular and so $Y$ is $M$ injective by (b). By [1, Proposition 16.13] $Y$ is $U$-injective. Thus, by Lemma $3, U$ is an SI-module. Moreover, since $\operatorname{Soc}(U)=0$ we can easily verify that every simple module in $\sigma[U]$ is $U$-injective. Hence, the same argument as that used for proving (i) $\Rightarrow$ (ii) of Lemma 3 shows that every module in $\sigma[U]$ is semiprimitive. It follows from this and (b) that every countably generated module in $\sigma[U]$ is a direct sum of an $M$-projective module and an $M$-injective module. On the other hand, since $U$ is a submodule of $M$, every $M$-projective (respectively, $M$-injective) module is also $U$ projective (respectively, $U$-injective) by [1, Propositions 16.12 and 16.13]. Thus every countably generated module in $\sigma[U]$ is a direct sum of a $U$-projective module and a $U$-injective module. 
Now, by [5, Theorem 2.2] we may assume without loss of generality that $U$ has no non-zero fully invariant proper submodules and then $\sigma[U]$ is Morita-equivalent with Mod- $T$ for some right SI-domain $T$ which is not a division ring. It follows that every countably generated right $T$-module is a direct sum of a projective module and an injective module. By [4], $T$ must be a division ring, a contradiction. Thus $S$ has to be an essential submodule of $M$ and hence $M / S$ is semisimple by Lemma 3 . This shows furthermore that every module in $\sigma[M]$ has an essential socle.

Next we show that every cyclic semiprimitive module $X$ in $\sigma[M]$ is semisimple. Since $X / \operatorname{Soc}(X)$ is $M$-singular, by Lemma $3, X / \operatorname{Soc}(X)$ is semisimple, in particular $X / \operatorname{Soc}(X)$ has finite (composition) length. Suppose on the contrary that $X$ is not semisimple. Then, $X$ is not Artinian. (Note that in this case, $X$ is semisimple if and only if $X$ is Artinian.) Hence $\operatorname{Soc}(X)$ is infinitely generated. Obviously, there exist finitely many elements $x_{1}, x_{2}, \ldots, x_{n}$ in $X$ such that

$$
X=x_{1} R+\cdots+x_{n} R+\operatorname{Soc}(X)
$$

and each $\left(x_{i} R+\operatorname{Soc}(X)\right) / \operatorname{Soc}(X)$ is simple. Put $U=\left(x_{1} R+x_{2} R+\cdots+x_{n} R\right) \cap$ $\operatorname{Soc}(X)$. Then $\operatorname{Soc}(X)=U \oplus V$ for some submodule $V$ of $\operatorname{Soc}(X)$. It follows that

$$
X=\left(x_{1} R+\cdots+x_{n} R\right) \oplus V
$$

and so $V_{R}$ is of finite length and hence the socle of one of the $x_{i} R$ 's must be infinitely generated. We may assume that $\operatorname{Soc}\left(x_{1} R\right)$ is infinitely generated. Put $T=x_{1} R$. Then we can easily check that also every finitely generated submodule of $\operatorname{Soc}(T)$ is a direct summand of $T$, and it follows from this that $J(T)=0$, that is, $T$ is semiprimitive. Moreover $T / \operatorname{Soc}(T)$ is simple. Since $T$ is semiprimitive, $T=W \oplus V$ by hypothesis, where $W$ is $M$-injective and $V$ is $M$-projective. Since $T \in \sigma[M]$ we see from [1, Proposition 16.13] that $W$ is quasi-injective and from [1, Proposition 16.12] that $V$ is quasi-projective. Since $W / \operatorname{Soc}(W)$ is semisimple by Lemma $3, W / \operatorname{Soc}(W)$ has finite length. By [7, Lemma 1.1], $W$ must have finite length; $W$ is even the finite direct sum of simple modules. Thus in considering $T$ we may, without loss of generality, assume that $T=V$ and so $T$ is $M$-projective, in particular $T$ is quasi-projective.

Now let $\operatorname{Soc}(T)=\bigoplus_{i \in I} T_{i}$ where $I$ is an infinite index set and each $T_{i}$ is a minimal submodule of $T$. We may assume that $I$ contains the set $\mathbb{N}$ of natural numbers and consider the submodule $U=\bigoplus_{i \in \mathbb{N}} T_{i}$ of $\operatorname{Soc}(T)$. Let $S=\operatorname{End}_{R}(T)$. By the assumptions on $T$, that is $T$ is semiprimitive and quasi-projective, we can use [1, Proposition 17.11] to see that $J(S)=0$, in particular $S$ does not contain non-zero one-sided nilpotent ideals and so $S$ is semiprime. We use this to consider $U$ as below. Since each minimal submodule $T_{i}$ of $U$ is a direct summand of $T$, for each $T_{i}$ there exists an idempotent 
$f_{i} \in S$ such that $f_{i} T=T_{i}$. Moreover, since $f_{i}$ is the identity of $\operatorname{End}_{R}\left(f_{i} T\right)$, it is easy to see that $\operatorname{End}_{R}\left(f_{i} T\right) \cong f_{i} S f_{i}$, that is, $f_{i} S f_{i}$ is a division ring and therefore for such idempotents $f_{i}, f_{i} S$ are minimal right ideals of $S$. Then by a standard argument we can show that there exists a system $\left\{e_{i}\right\}_{i \in \mathrm{N}}$ of orthogonal idempotents in $S$ such that each $e_{i} T$ is minimal and

$$
U=\bigoplus_{i \in \mathrm{N}} e_{i} T
$$

Now we divide $\mathbb{N}$ into two infinite subsets $\mathbb{N}_{1}$ and $\mathbb{N}_{2}$ such that $\mathbb{N}=\mathbb{N}_{1} \cup \mathbb{N}_{2}$ and $\mathbb{N}_{1} \cap \mathbb{N}_{2}=\emptyset$. Put

$$
U_{1}=\bigoplus_{i \in \mathbb{N}_{1}} e_{i} T \text { and } U_{2}=\bigoplus_{j \in \mathbb{N}_{2}} e_{j} T .
$$

For each finite subset $F_{\alpha}$ of $\mathbb{N}_{1}$ put $e_{\alpha}=\sum_{i \in F_{\alpha}} e_{i}$. Then $e_{\alpha}$ is an idempotent and so $T=e_{\alpha} T \oplus V_{\alpha}$ with $V_{\alpha}=\left(1-e_{\alpha}\right) T$. It follows that $U_{2} \subseteq V_{\alpha}$. Let $V=\bigcap V_{\alpha}$ where $\alpha$ runs through all indices of finite subsets $F_{\alpha}$ of $\mathbb{N}_{1}$. Then we have $U_{2} \subseteq V$ and

$$
\bar{T}:=T / V=\left(\left(\bigoplus_{i \in F_{\alpha}} e_{i} T\right)+V\right) / V \oplus V_{\alpha} / V .
$$

If we denote by $\bar{U}_{1}$ the image of $U_{1}$ in $\bar{T}$, then $\bar{U}_{1} \cap J(T)=\overline{0}$, in particular, $\bar{T} / J(T)$ has an infinitely generated socle. By hypothesis, we have

$$
\bar{T} / J(\widetilde{T})=\widetilde{P} \oplus \widetilde{Q}
$$

where $\widetilde{P}$ is $M$-projective and $\widetilde{Q}$ is $M$-injective. As we previously saw, the socle of $\bar{T} / J(T)$ is infinitely generated. Furthermore, since $T / \operatorname{Soc}(T)$ is simple, it is easy to see that $\widetilde{Q} / \operatorname{Soc}(\widetilde{Q})$ is simple or zero. If $\widetilde{Q} / \operatorname{Soc}(\widetilde{Q})$ is zero, then $\operatorname{Soc}(\widetilde{Q})=\widetilde{Q}$ is of finite length, because $\tilde{Q}$ is cyclic. If $\widetilde{Q} / \operatorname{Soc}(\tilde{Q})$ is simple, $\operatorname{Soc}(\tilde{Q})$ must be of finite length by $[7$, Lemma 1.1]. In any case, $\widetilde{Q}$ has finite length and hence the socle of $\widetilde{P}$ is infinitely generated.

Finally, let $\bar{Q}$ be the inverse image of $\widetilde{Q}$ in $\bar{T}$ and $Q$ the inverse image of $\bar{Q}$ in $T$. Then $V \subseteq Q$, in particular, $\operatorname{Soc}(Q)$ is infinitely generated, since $U_{2} \subseteq \operatorname{Soc}(Q)$. Moreover, since $T / Q \cong \widetilde{P}$ is $M$-projective, by $[13,18.3(\mathrm{~d})$-(h)] the exact sequence

$$
0 \rightarrow Q \hookrightarrow T \rightarrow T / Q \rightarrow 0
$$

splits, that is,

$$
T=P \oplus Q
$$


for some submodule $P$ of $T$ with $P \cong \widetilde{P}$. Since $T$ is cyclic, we must have by (1) that $P \neq \operatorname{Soc}(P)$ and $Q \neq \operatorname{Soc}(Q)$. Hence $T / \operatorname{Soc}(T) \cong P / \operatorname{Soc}(P) \oplus Q / \operatorname{Soc}(Q)$ has length at least 2, a contradiction. Thus $X$ must be Artinian, and so $X$ is semisimple.

Now let $N$ be a semiprimitive module in $\sigma[M]$. Then every minimal submodule of $N$ is a direct summand of $N$. If $N$ is not semisimple, then there exists a cyclic submodule $X$ of $N$ such that $X$ is not semisimple. Moreover we can check easily that any minimal submodule of $X$ is also a direct summand of $X$. But $\operatorname{Soc}(X)$ is essential in $X$. Hence $J(X)=0$, that is, $X$ is semiprimitive. Therefore $X$ is semisimple as shown above, a contradiction. Thus $N$ is semisimple, proving (c).

(c) $\Rightarrow$ (a). Assume (c) and let $N$ be a semiprimitive module in $\sigma[M]$. By (c), $N$ is semisimple. Hence we can write $N$ in the form

$$
N=\left(\bigoplus_{i \in I} N_{i}\right) \oplus\left(\bigoplus_{j \in J} N_{j}\right)
$$

where each $N_{i}(i \in I)$ is simple and $M$-nonsingular and each $N_{j}(j \in J)$ is simple and $M$-singular. Since $M$ is a quasi-projective SI-module, it follows that $\bigoplus_{i \in I} N_{i}$ is $M$-projective and $\bigoplus_{j \in J} N_{j}$ is $M$-injective. This means that we have (a).

The proof of Theorem 4 is complete.

Now we prove Theorem 2. The implication (i) $\Rightarrow$ (ii) is clear. From (ii) it follows that $R$ is semiprimary and right SI by Theorem 4. By [3, Proposition 3.5] $R$ is then left SI, that is, we have (iii). The implication (iii) $\Rightarrow$ (i) is also clear by Theorem 4 . The equivalence (iii) $\Leftrightarrow$ (iv) follows from the symmetry of (iii). Thus the proof is complete.

Finally we note that it is easy to find a ring as in Theorem 2 which is neither left nor right Artinian. For example, if $\mathbb{Q}$ is the field of rational numbers and $\mathbb{Q}(x)$ is the field of fractions of the polynomial ring $\mathbb{Q}[x]$. Then the ring

$$
\left(\begin{array}{cc}
\mathbb{Q} & \mathbb{Q}(x) \\
0 & \mathbb{Q}
\end{array}\right)
$$

is a semiprimary SI-ring which is neither right nor left Artinian.

\section{REFERENCES}

[1] F.W. Anderson and K.R. Fuller, Rings and Categories of Modules (Springer-Verlag, Berlin, Heidelberg, New York, 1974).

[2] C. Faith, Algebra II: Ring theory (Springer-Verlag, Berlin, Heidelberg, New York, 1976).

[3] K.R. Goodearl, Singular torsion and splitting properties, Amer. Math. Soc. Memoirs 124 (American Mathematical Society, 1972). 
[4] D.V. Huynh and P. Dan, 'A result on Artinian rings', Math. Japon. 35 (1990), 699-702.

[5] D.V. Huynh and R. Wisbauer, 'A structure theorem for SI-modules', Glasgow Math. J. 34 (1992), 83-89.

[6] D.V. Huynh and R. Wisbauer, 'Self-projective modules with $\pi$-injective factor modules', J. Algebra 153 (1992), 13-21.

[7] D.V. Huynh, N.V. Dung and R. Wisbauer, 'On modules with finite uniform and Krull dimension', Arch. Math. 57 (1991), 122-132.

[8] G.O. Michler and O.E. Villamayor, 'On rings whose simple modules are injective', $J$. Algebra 25 (1973), 185-201.

[9] S.H. Mohamed and B.J. Müller, Continuous and discrete modules, London Math. Soc. Lecture Note Series 147 (London Mathematical Society, 1990).

[10] K. Oshiro, 'Lifting modules, extending modules and their applications to QF-rings', Hokkaido Math. J. 13 (1984), 310-338.

[11] B.L. Osofsky, 'Rings all of whose finitely generated modules are injective', Pacific J. Math. 14 (1964), 645-650.

[12] B.L. Osofsky and P.F. Smith, 'Cyclic modules whose quotients have all complement submodules direct summands', J. Algebra 139 (1991), 342-354.

[13] R. Wisbauer, Foundations of module and ring theory (Gordon and Breach, Reading, 1991).

Department of Mathematics

Okayama University

Okayama 700

Japan

Department of Mathematics

Busan National University

Busan 609-735

South Korea

\author{
Institute of Mathematics \\ PO Box 631 Boho \\ Hanoi \\ Vietnam
}

